\title{
Implementation of Six-axis Inertial Measurement Unit on a Stretchable Platform Using "Cut-and-Paste" Method for Biomedical Applications
}

\author{
Kangil Kim and Sangmin Lee \\ Department of Biomedical Engineering, Kyung Hee University, Yongin, Gyeonggi 17104, Republic of Korea
}

(Received April 23, 2018; accepted March 13, 2019)

Keywords: epidermal electronics, inertial measurement unit (IMU), stretchable platform, cut-and-paste

Wearable devices that can process and transmit signals from the human body have the potential to provide valuable biometric data for mobile health (mHealth) and human-machine interface (HMI). Among recent advancements in flexible and stretchable electronics, epidermal electronic devices have shown major breakthroughs in wearable device research fields. Epidermal systems, such as tattoo- and patch-type stretchable systems, have conformity to the human skin, which can be deformed with the skin without detachment for fracture. In this paper, the implementation of a six-axis inertial measurement unit (IMU) on a stretchable platform for applications in wearable electronics is presented. The proposed device is implemented using circuit elements on a patchlike substrate to collect accurate data from the human body. To achieve flexible and stretchable characteristics of the device, a serpentine interconnection is adopted between the circuit elements. Instead of a microfabrication process, a low-cost, dry, and freeform "cut-and-paste" method that uses a thin metal sheet and a cutting tool to fabricate complex serpentine interconnections is used to fabricate the substrate. The performance characteristics of the IMU using the proposed method are demonstrated for future application in highly accurate biosensors.

\section{Introduction}

Wearable devices that can process and transmit signals from the human body have the potential to provide valuable biometric data for mobile health (mHealth), ${ }^{(1-3)}$ consumer electronics, ${ }^{(4,5)}$ and human-machine interface (HMI). ${ }^{(6-8)}$ In particular, recent wearable medical devices focus on efficient skinlike sensing platforms for medical monitoring, imaging, and diagnostics..$^{(9,10)}$ These skinlike sensing platforms, also known as epidermal electronic devices, have soft form factors that allow close attachment to the human skin, which significantly increases the signal-to-noise ratio (SNR). ${ }^{(11)}$ In addition, with the inherent flexible and stretchable characteristics of these platforms, recent research studies emphasize the development of highly configurable and scalable epidermal electronic devices

*Corresponding author: e-mail: sangmlee@khu.ac.kr https://doi.org/10.18494/SAM.2019.2259 
for diverse biomedical applications. Generally, most of the recently developed systems are implemented using silicon-based integrated circuits (ICs) for signal conditioning, which provide an incomparable computational performance at a low power than any other organic/ inorganic material-based circuits. ${ }^{(12,13)}$ However, as a trade-off, there are possible causes of a large mechanical discrepancy in the system-skin interface between hard silicon-based ICs and the soft human skin. Therefore, to resolve this issue, a hybrid solution using soft and hard electronic parts can be adopted, where flexible/stretchable interconnection wires are used for soft biointerfaces, and hard electronic ICs are used for computation, signal conditioning, and data transmission.

To establish high-fidelity system-skin interfaces, serpentine-structured metal interconnection wires are used to connect silicon-based sensors and ICs, which can be implemented by printing methods or semiconductor microfabrication processes. Owing to the serpentine structure of the interconnection wires, even if the system is elongated or bent, the stress on the wires can be minimized to avoid resistance variations, breakage or signal losses between the sensors and the ICs. Therefore, achieving the robustness of the interconnection parts is a fundamental enabling technology for system-level implementation of epidermal stretchable/flexible electronic devices. However, the typical constraints for the implementation of these devices are encountered by the fabrication processes. Kim et al. reported a flexible e-skin sensor interface using a flexible printed circuit board (PCB) implementation method. ${ }^{(14)}$ However, the method requires photomask fabrication for lithography and multiple steps of lamination and metal electroplating. Also, many research groups have reported on using semiconductor fabrication processes for implementing a serpentine-structured interconnection. However, the microfabrication using cleanroom facilities is usually highly expensive and time-consuming owing to high-vacuum deposition/etching processes, high-cost masks for photolithography processes, toxic chemical wet etching processes, and high-temperature annealing processes. Although its effectiveness and reliability are well known to achieve micropatterns that minimize the footprint for miniaturized system implementations, such a process is costly and time-consuming.

In this paper, an epidermal flexible/stretchable six-axis inertial measurement unit (IMU) is presented for biomedical applications such as dependency care, musculoskeletal disorders, and joint kinematics, and for monitoring daily routines. ${ }^{(15-18)}$ The importance of human body inertia data is growing in the research of age-related kinematic changes, the identification of neurodegenerative diseases, the assessment of rehabilitation evolution, and the assessment of sports biomechanics. The proposed system consists of hard silicon-based ICs with serpentinestructured interconnection wires and can be laminated on any part of the human skin with high conformity, which enables enhanced SNR and accuracy, despite the skin deformation. The system is implemented using the fully modularized structure consisting of a commercialized six-axis inertial sensor, a microcontroller unit (MCU), and passive electronic components, which measures continuous biometric information with ease. To implement the system, the "cut-and-paste" manufacturing method, ${ }^{(19)}$ which does not require cleanroom microfabrication processes including high-vacuum, high-temperature, and plasma-utilized dry and highly toxic chemical wet processes, is used. The design, fabrication method, and evaluation results will be described in detail in the following sections. The results in this paper can be extended to develop an advanced multisensing platform. 


\section{Methods}

\subsection{Design}

The structural design of a patch-type, epidermal six-axis IMU on a flexible/stretchable platform is shown in Fig. 1. The proposed epidermal stretchable system consists of conventional hard silicon-based electronics, such as a six-axis inertial sensor (three-axis accelerometer/ three-axis gyroscope) (MPU6050, InvenSense, USA), a $16 \mathrm{MHz}$ MCU (ATmega128, Microchip Technology Inc., USA), and passive circuit components for measuring the six-axis motion. Serpentine-structured copper $(\mathrm{Cu})$ wires are used to assemble and interface hard electronic devices, which have elongation capabilities under various deformations. The entire system is encapsulated by a soft adhesive film (Tegaderm, 3M, USA), which provides flexibility and stretchability. The system is implemented on the basis of the modular concept, indicating that functional building blocks can be easily replaced or added depending on the target application. By maintaining the signal conditioning and wired/wireless transmission parts, additional functions can be equipped by adding sensors, such as strain gauges for respiration, electrodes for electrocardiography (ECG), or light-emitting diodes (LEDs) and photodetectors for pulse oximetry. The overall dimensions of the proposed system are 40 and $120 \mu \mathrm{m}$, and the thickness is less than $100 \mu \mathrm{m}$ (excluding the ICs).

The block diagram of the epidermal six-axis IMU is shown in Fig. 2. The six-axis inertial sensor and MCU send and receive signals in a two-wire serial interface (TWI). The TWI communication is a serial communication method, in which up to 128 devices can be connected with only two bus signals (SCL and SDA) to exchange data. The inertial signals from the sensor are digitized with the embedded analog-to-digital converter (ADC), and the encoded data are displayed using universal synchronous/asynchronous receiver/transmitter (USART) communication by the MCU.

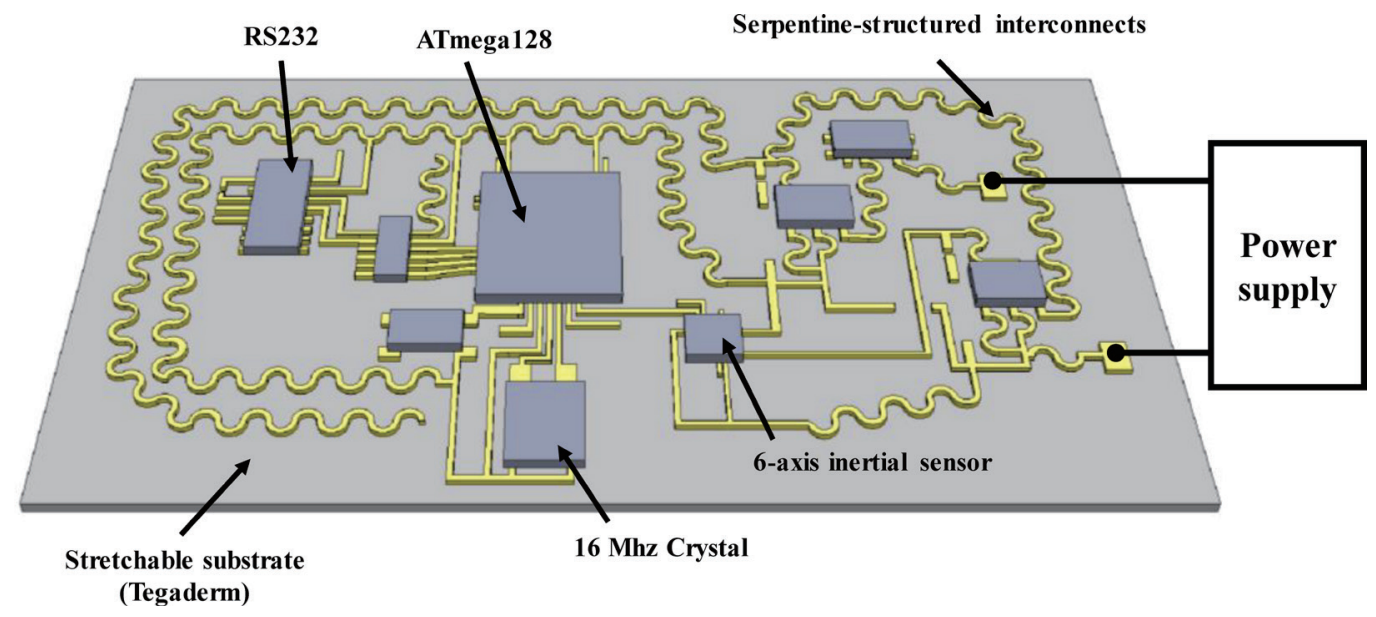

Fig. 1. (Color online) Schematic of the structural design of the epidermal six-axis IMU. 


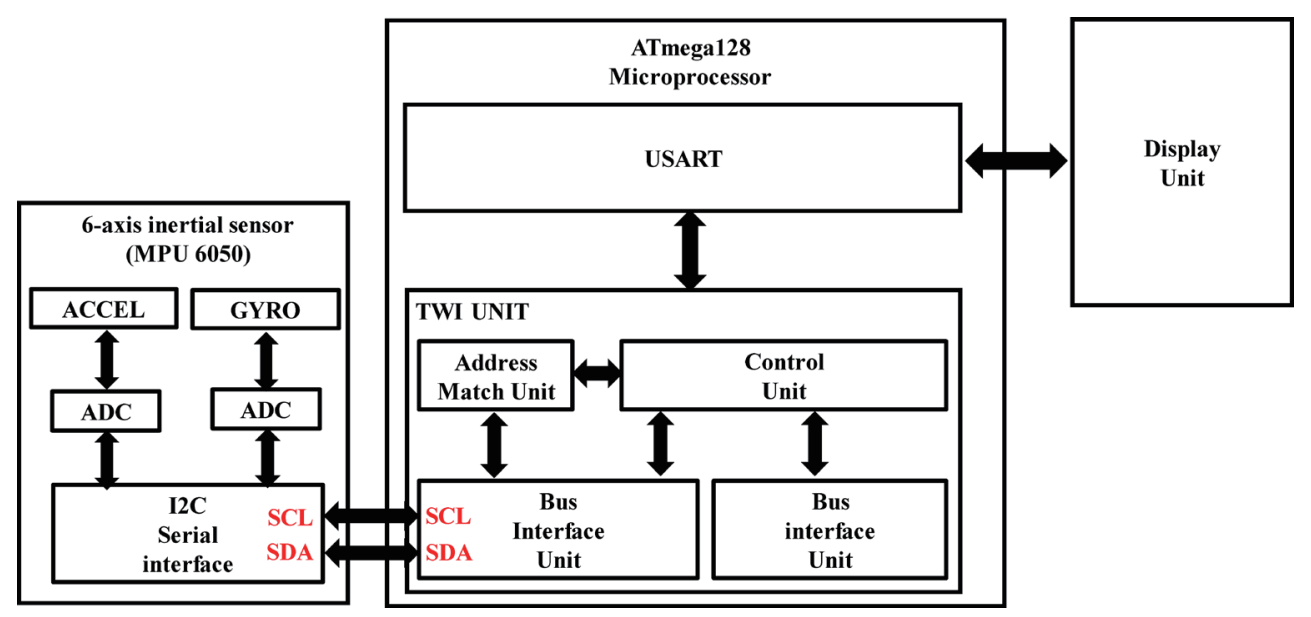

Fig. 2. (Color online) Block diagram of the epidermal six-axis IMU.

\subsection{Implementation process and results}

To implement the stretchable six-axis IMU on a flexible/stretchable polymeric surface, a "cut-and-paste" manufacturing process is utilized, as shown in Fig. 3. The process uses direct computer-aided design (CAD) programs to realize the rapid prototyping of the miniaturized system on an unfamiliar substrate without photomasks and semiconductor microfabrication processes. To implement the epidermal stretchable system, the serpentine-structured interconnection wires and bonding pads for silicon IC alignment are fabricated. After designing the footprints of the system using the CAD tool, bench-top programmable cutting equipment (Silhouette Cameo ${ }^{\circledR}$, Silhouette America) is used to define the interconnection wires and bonding pads. The minimum pattern size that can be fabricated by the equipment is approximately $200 \mu \mathrm{m}$, which is sufficient for implementing a miniaturized system.

First, as shown in Fig. 3(a), an 18- $\mu \mathrm{m}$-thick Cu foil (Copper 110 Annealed, OnlineMetals, USA) was laminated on a thermal release tape (TRT) (Haeun Chemtec Co., Ltd., Republic of Korea). The Cu-foil-laminated TRT is adhered to a cutting mat, and the equipment starts to define the designed patterns programmed from the CAD tool [Fig. 3(b)]. The cutting depth is programmed only to cut the $\mathrm{Cu}$ foil layer by manipulating the height of the cutting knife. Then, a glass substrate adhered to a sequential Kapton film and a water-soluble tape (WST) layer is prepared, as shown in Fig. 3(c). The patterned Cu-foil-laminated TRT layer is bonded to the prepared glass substrate with the $\mathrm{Cu}$ foil side facing down. After removing the $\mathrm{Cu}-$ foil-laminated TRT from the cutting mat, every residual area of the $\mathrm{Cu}$ foil is peeled off using tweezers [Fig. 3(d)]. The bonded substrate is heated on a hot plate at $120^{\circ} \mathrm{C}$ for $30 \mathrm{~s}$ to remove the TRT layer. After removing the TRT layer, solder paste (lead-free, ChipQuik) is applied to the bonding pads of the patterned $\mathrm{Cu}$ foil, where silicon-based ICs will be located. Electronic components, such as a six-axis digital accelerometer, a $16 \mathrm{MHz} \mathrm{MCU}$, passive circuit elements, and interconnection bridges, are placed on top of the solder paste, and heated on the hot plate at $230{ }^{\circ} \mathrm{C}$ for $3 \mathrm{~min}$ until the components are completely fixed [Fig. 3(e)]. Then a thin adhesive 


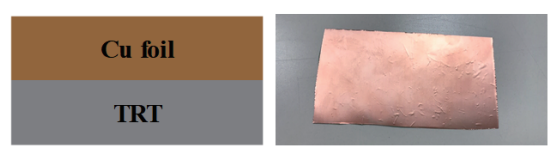

(a) Cu foil laminated on TRT

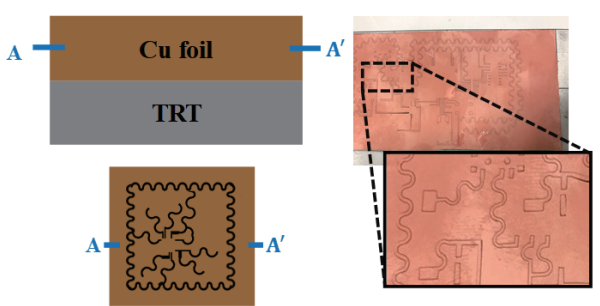

(b) Cu foil patterning

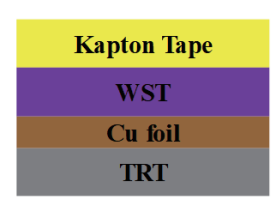

(c) Transfer of $\mathrm{Cu}$ circuit on WST/Kapton tape

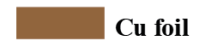

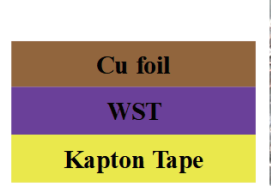

(d) TRT removal and soldering chip components on the $\mathrm{Cu}$ circuit

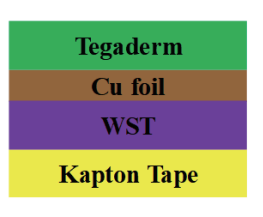

(e) Transfer to stretchable substrate (Tegaderm) and WST/Kapton tape removal

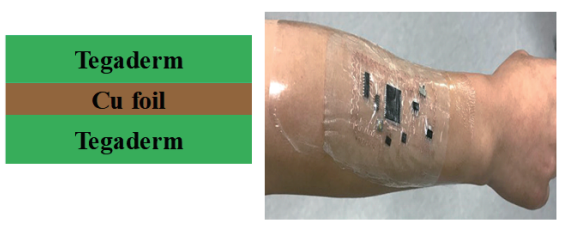

(f) Encapsulation of circuitry

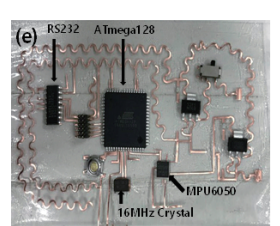

Kapton Tape

Tegaderm

Fig. 3. (Color online) Schematic of the "cut-and-paste" fabrication process.

film, Tegaderm, is attached to the circuitry for top-layer encapsulation. The WST layer is dissolved after peeling off the Kapton tape from the glass substrate to leave only the electronic components and serpentine-structure $\mathrm{Cu}$ interconnection wires. After covering the bottom layer using Tegaderm, the epidermal six-axis IMU on a stretchable platform is implemented, as shown in Fig. 3(f).

The implemented stretchable six-axis IMU is attached to the right arm as shown in Fig. 3(f). As reported previously, ${ }^{(15)}$ the stress applied to the serpentine-structured interconnection wire during elongation is less. Therefore, the modulus of the implemented device depends on the stiffness of Tegaderm (approximately 7.4 MPa), which is similar to that of the human skin (0.32-4 MPa). The electronic components are well aligned and attached when the device is stretched up to $20 \%$. The flexibility and stretchability of the substrate indicate that the implemented device can be utilized for the human body including the wrists, ankles, neck, and abdominal region, which are common parts for monitoring the motion for dependency care and clinical diagnosis.

\section{Performance Evaluation}

To determine the design of the serpentine-structured interconnection wire, the experiment is conducted with varying parameters of the serpentine structure. As described in Fig. 4 and Table 1, the curvature radius $(r)$, rotation angle $(\theta)$, and pattern width $(w)$ are determined to fabricate the 


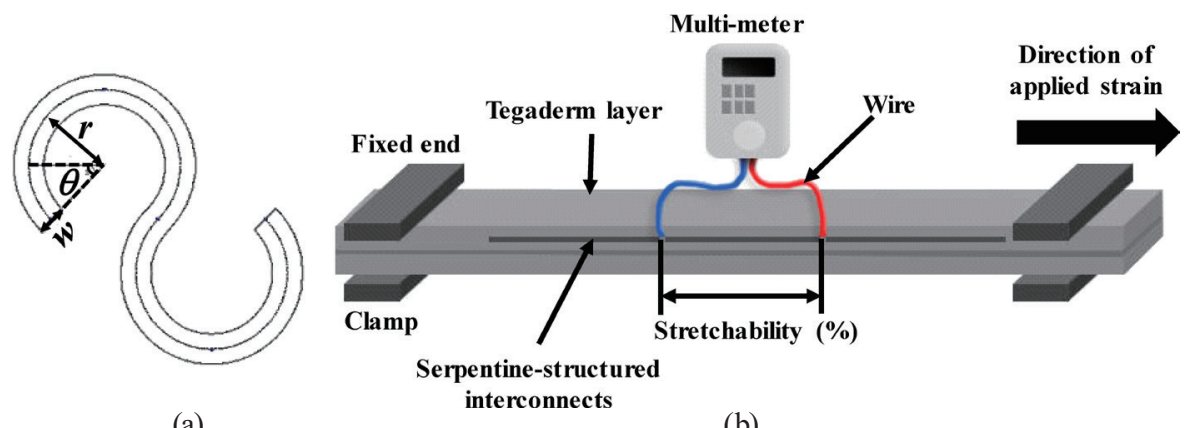

(a)

(b)

Fig. 4. (Color online) (a) Design constraints for serpentine-structured interconnection wire. (b) Experimental setup (resistance variation vs stretchability).

Table 1

(Color online) Summarized results of resistance variation of serpentine-structured interconnection.

\begin{tabular}{|c|c|c|c|c|}
\hline $\begin{array}{l}\text { Design } \\
{\left[w(\mathrm{~mm}), r(\mathrm{~mm}), \theta\left(^{\circ}\right)\right]}\end{array}$ & $\begin{array}{l}\text { Initial length } \\
(\mathrm{mm})\end{array}$ & $\begin{array}{l}\text { Initial resistance } \\
\qquad(\mathrm{m} \Omega)\end{array}$ & $\begin{array}{l}\text { Maximum resistance variation } \\
(\%)(20 \% \text { elongation })\end{array}$ & Image \\
\hline$w=300, r=1, \theta=0$ & 135 & 206 & 1.319 & \\
\hline$w=300, r=1, \theta=45$ & 96 & 642 & 0.377 & \\
\hline$w=300, r=1.25, \theta=0$ & 123 & 642 & 1.569 & \\
\hline$w=300, r=1.25, \theta=45$ & 88 & 428 & 1.067 & \\
\hline$w=300, r=1.5, \theta=0$ & 118 & 200 & 4.948 & \\
\hline$w=300, r=1.5, \theta=45$ & 90 & 688 & 1.039 & \\
\hline$w=500, r=1, \theta=0$ & 147 & 123 & 0.291 & \\
\hline$w=500, r=1, \theta=45$ & 105 & 254 & 0.816 & \\
\hline$w=500, r=1.25, \theta=0$ & 132 & 159 & 1.922 & \\
\hline$w=500, r=1.25, \theta=45$ & 119 & 315 & 0.940 & trin \\
\hline$w=500, r=1.5, \theta=0$ & 147 & 162 & 1.700 & \\
\hline$w=500, r=1.5, \theta=45$ & 103 & 318 & 1.119 & \\
\hline$w=700, r=1, \theta=0$ & 158 & 110 & 0.589 & nun \\
\hline$w=700, r=1, \theta=45$ & 113 & 217 & 1.207 & न \\
\hline$w=700, r=1.25, \theta=0$ & 141 & 116 & 1.173 & Hurñ \\
\hline$w=700, r=1.25, \theta=45$ & 107 & 220 & 2.318 & -5888080808808G \\
\hline$w=700, r=1.5, \theta=0$ & 140 & 119 & 1.303 & Hoverar \\
\hline$w=700, r=1.5, \theta=45$ & 109 & 222 & 3.686 & 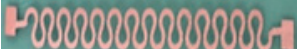 \\
\hline
\end{tabular}


device, and the rate of change in resistance at elongation is measured. The results show that the rate of change in resistance has little difference, indicating that the serpentine structures do not affect signal attenuation or performance degradation.

In this study, a patch-type six-axis IMU was implemented using a "cut-and-paste" method. Since the circuit is encapsulated with a stretchable substrate and its interconnections have serpentine structures, it can be adhered closely to the body. Also, there is no distortion of the output signal even if it is stretched. Three experiments were conducted to confirm the normal operation of the patch-type six-axis IMU. First, as shown in Fig. 5(a), after setting the sensitivity of the three-axis acceleration sensor to $2 \mathrm{~g}$, an experiment was performed so that the sensor showed 0 and $1 \mathrm{~g}$ for each axis. It can be confirmed that the $x$-, $y$-, and $z$-axes operate normally. Second, in the case of a gyro sensor, the roll axis has a Euler angle in the range of -180 to $+180^{\circ}$, the pitch axis has a Euler angle in the range of -90 to $+90^{\circ}$, and the yaw axis has a Euler angle of -180 to $+180^{\circ}$. As can be seen in Fig. 5(b), the appropriate waveform appears for each axis. Finally, in Fig. 5(c), an experiment is conducted by applying the arbitrary motion to the patch-type six-axis IMU attached to the arm. From the results, the real-time, continuous biometric motion data of the human body can be successfully monitored by personalized mobile devices and network servers to log the activity of a personnel in a long term.

(a)
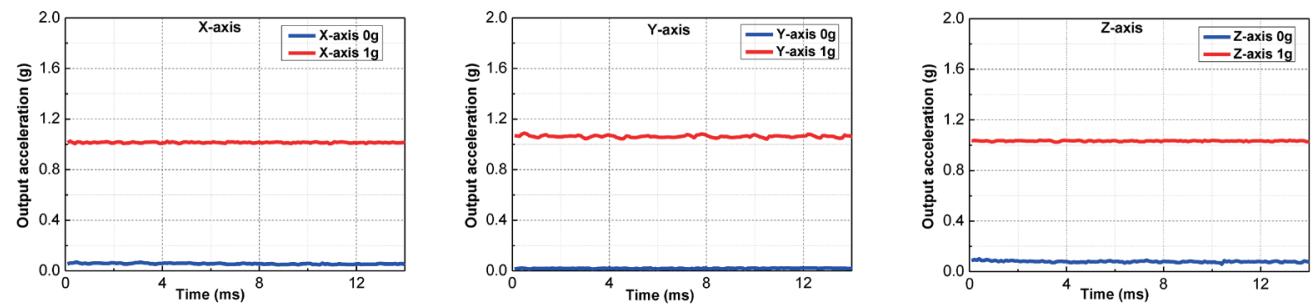

(b)
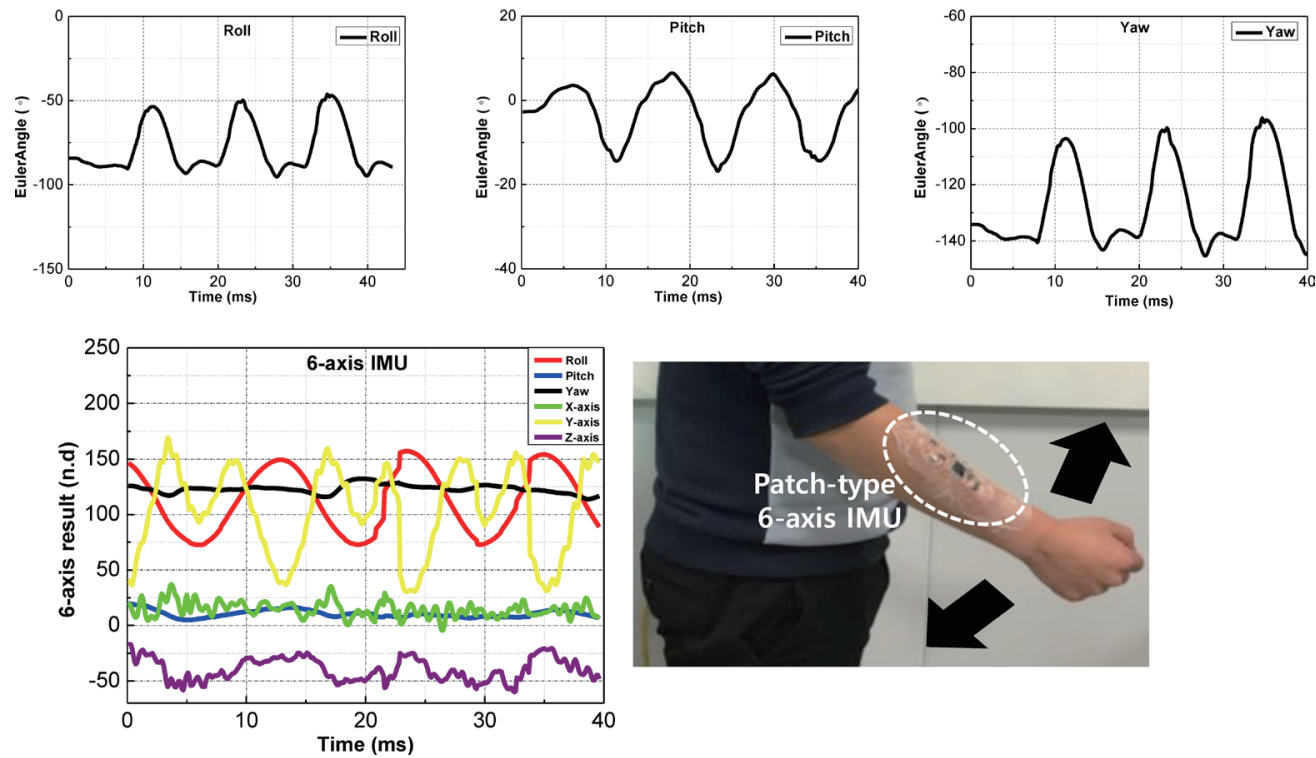

Fig. 5. (Color online) Performance evaluation of the epidermal six-axis IMU: (a) three-axis acceleration, (b) three-axis rotation, and (c) arbitrary motion. 


\section{Conclusion}

In this paper, the implementation of a patch-type six-axis IMU for applications in wearable electronics is presented. The proposed device is implemented using circuit elements on a patchlike substrate to collect accurate data from the human body. To achieve flexible and stretchable characteristics of the device, a serpentine interconnection is adopted between the circuit elements. Instead of a microfabrication process, the "cut-and-paste" method, which uses a thin metal sheet and a cutter tool to fabricate complex serpentine interconnections, is used to fabricate the substrate. The preliminary implementation and the performance characteristics of the IMU using the proposed method are shown for future application.

\section{Acknowledgments}

This work was supported by the National Research Foundation of Korea (NRF) grant funded by the Korea government [MSIP; Ministry of Science, ICT \& Future Planning (No. NRF2017R1C1B5017561)].

\section{References}

1 D. Kim, N. Lu, R. Ma, Y. Kim, R. Kim, S. Wang, J. Wu, S. M. Won, H. Tao, A. Islam, K. J. Yu, T. Kim, R. Chowdhury, M. Ying, L. Xu, M. Li, H. Chung, H. Keum, M. McCormick, P. Liu, Y. Zhang, F. G. Omenetto, Y. Huang, T. Coleman, and J. A. Rogers: Science 333 (2011) 838.

2 H. Lee, T. K. Choi, Y. B. Lee, H. R. Cho, R. Ghaffari, L. Wang, H. J. Choi, T. D. Chung, N. Lu, T. Hyeon, S. H. Choi, and D. Kim: Nat. Nanotechnol. 11 (2016) 566.

3 A. Miyamoto, S. Lee, N. F. Cooray, S. Lee, M. Mori, N. Matsuhisa, H. Jin, L. Yoda, T. Yokota, A. Itoh, M. Sekino, H. Kawasaki, T. Ebihara, M. Amagai, and T. Someya: Nat. Nanotechnol. 12 (2017) 907.

4 C. Kim, H. Lee, K. H. Oh, and J. Sun: Science 353 (2016) 411.

5 Y. H. Jung, J. Lee, Y. Qiu, N. Cho, S. J. Cho, H. Zhang, S. Lee, T. J. Kim, S. Gong, and Z. Ma: Adv. Funct. Mater. 26 (2016) 4635.

6 Y. Liu, J. J. S. Norton, R. Qazi, Z. Zou, K. R. Ammann, H. Liu, L. Yan, P. L. Tran, K. Jang, J. W. Lee, D. Zhang, K. A. Kilian, S. H. Jung, T. Bretl, J. Xiao, M. J. Slepian, Y. Huang, J. Jeong, and J. A. Rogers: Sci. Adv. 2 (2016) e1601185.

7 J. Jeong, W. Yeo, A. Akhtar, J. J. S. Nrton, Y. Kwack, S. Li, S. Jung, Y. Su, W. Lee, J. Xia, H. Cheng, Y. Huang, W. Choi, T. Bretl, and J. A. Rogers: Adv. Mater. 25 (2013) 6839.

8 S. Lim, D. Son, J. Kim, Y. B. Lee, J. Song, S. Choi, D. J. Lee, J. H. Kim, M. Lee, T. Hyeon, and D. Kim: Adv. Funct. Mater. 25 (2015) 375.

9 S. L. Swisher, M. C. Lin, A. Liao, E. J. Leeflang, Y. Khan, F. J. Pavinatto, K. Mann, A. Naujokas, D. Young, S. Roy, M. R. Harrison, A. C. Arias, V. Subramanian, and M. M. Maharbiz: Nat. Commun. 6 (2015) 6575.

10 R. C. Webb, A. P. Bonifas, A. Behnaz, Y. Z. Ki, K. J. Yu, H. Cheng, M. Shi, Z. Bian, Z. Liu, Y. Kim, W. Yeo, J. S. Park, J. Song, Y. Li, Y. Huang, A. M. Gorbach, and J. A. Rogers: Nat. Mater. 12 (2013) 938.

11 M. Kaltenbrunner, T. Sekitani, J. Reeder, T. Yokota, K. Kuribara, T. Tokuhara, M. Drack, R. Schwodiauer, I. Graz, S. Bauer-Gogonea, S. Bauer, and T. Someya: Nature 499 (2013) 458.

12 Q. Cao, H.-S. Kim, N. Pimparkar, J. P. Kulkarni, C. Wang, M. Shim, K. Roy, M. A. Alam, and J. A. Rogers: Nature 454 (2008) 495.

13 B. Crone, A. Dodabalapur, Y.-Y. Lin, R. Filas, Z. Bao, A. LaDuca, R. Sarpeshkar, H. Katz, and W. Li: Nature 403 (2000) 521.

14 S. Kim, Y. Lee, J. Park, S. Kim, H. Chae, H. Ko, and J. J. Kim: Sensors 18 (2018) 78.

15 D. Rodriquez-Martin, C. Perez-Lopez, A. Sama, J. Capestany, and A. Catala: Sensors 13 (2013) 14079.

16 X. Yan, H. Le, A. R. Li, and H. Zhang: Autom. Constr. 74 (2017) 2. 
17 K. Lebel, P. Boissy, H. Nguyen, and C. Duval: Biomed. Eng. Online 16 (2017) 56.

18 P. Rivera, E. Valarezo, M. Choi, and T. Kim: Int. J. Pharm. Med. Biol. Sci. 6 (2017) 114.

19 S. Yang, Y. C. Chen, L. Nicolini, P. Pasupathy, J. Sacks, B. Su, R. Yang, D. Sanchez, Y. F. Chang, P. Wang, and D. Schnyer: Adv. Mater. 27 (2015) 6423.

\section{About the Authors}

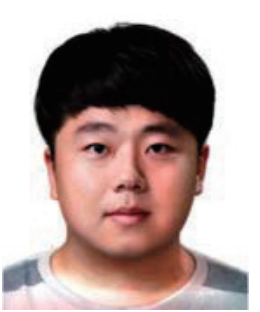

Kangil Kim is currently enrolled in an undergraduate course in the Department of Biomedical Engineering, Kyung Hee University, Yongin, Republic of Korea. His research interests are in the microfabrication of MEMS sensors and system implementation for biomedical applications.

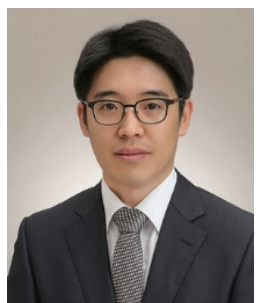

Sangmin Lee received his B.S, M.S, and Ph.D. degrees in the School of Electrical Engineering at Seoul National University, Republic of Korea, in 2005, 2007, and 2013, respectively. From 2013 to 2016, he worked as a research staff member at Samsung Advanced Institute of Technology (SAIT), Samsung Electronics, Inc, Republic of Korea. In 2016, he joined the Department of Biomedical Engineering, Kyung Hee University, Yongin, Republic of Korea, where he is currently an assistant professor. His main research interests are biomedical sensors and systems for applications in Internet of Things (IoT) devices. 